\title{
ON GLOBAL WELL-POSEDNESS FOR NONLINEAR SEMIRELATIVISTIC EQUATIONS IN SOME SCALING SUBCRITICAL AND CRITICAL CASES
}

\author{
KAZUMASA FUJIWARA, VLADIMIR GEORGIEV, AND TOHRU OZAWA
}

\begin{abstract}
In this paper, the global well-posedness of semirelativistic equations with a power type nonlinearity on Euclidean spaces is studied. In two dimensional $H^{s}$ scaling subcritical case with $1 \leq s \leq 2$, the local well-posedness follows from a Strichartz estimate. In higher dimensional $H^{1}$ scaling subcritical case, the local well-posedness for radial solutions follows from a weighted Strichartz estimate. Moreover, in three dimensional $H^{1}$ scaling critical case, the local well-posedness for radial solutions follows from a uniform bound of solutions which may be derived by the corresponding one dimensional problem. Local solutions may be extended by a priori estimates.
\end{abstract}

\section{INTRODUCTION}

In this paper, we consider the well-posedness of the following Cauchy problem:

$$
\begin{cases}i \partial_{t} u-(-\Delta)^{1 / 2} u=-i|u|^{p-1} u, & t \in \mathbb{R}, \quad x \in \mathbb{R}^{n}, \\ u(0)=u_{0}, & x \in \mathbb{R}^{n},\end{cases}
$$

where $n \geq 1, p>1, \Delta$ is the Laplacian, and $(-\Delta)^{1 / 2}=\mathfrak{F}^{-1}|\xi| \mathfrak{F}$ with the Fourier transform $\mathfrak{F}$.

Similar models can be connected with the simulations of neuroscience processes. Typical one is the cyclical alternation of REM (rapid eye movement) and NREM (non-rapid eye movement) sleep. See [ACGM]. The model of the alternation of REM and NREM sleep is starting from the classical $\mathrm{Ku}-$ ramoto model [Kur], having its origin in special type of Landau - Ginzburg model

$$
i \partial_{t} u-\mathcal{H} u=-i Q(u)
$$

2010 Mathematics Subject Classification. 35Q40, 35Q55.

Key words and phrases. semirelativistic equation, global well-posedness.

The first author was supported in part by the Japan Society for the Promotion of Science, Grant-in-Aid for JSPS Fellows no 16J30008 and Top Global University Project, Waseda University.

The second author was supported in part by Contract FIRB " Dinamiche Dispersive: Analisi di Fourier e Metodi Variazionali", 2012, by INDAM, GNAMPA - Gruppo Nazionale per l'Analisi Matematica, la Probabilita e le loro Applicazion and by Institute of Mathematics and Informatics, Bulgarian Academy of Sciences and Top Global University Project, Waseda University.

The third author was supported in part by Grant-in-Aid for Scientific Research (A) Number 26247014. 
where $\mathcal{H}$ is appropriate Hamiltonian operator and $Q(u)$ is appropriate cubic type nonlinearity. In this work we substitute the specific cubic nonlinearity $Q(u)$ by a self - interacting nonlinear term $|u|^{p-1} u$ and our goal is to implement the recent development of fractional quantum mechanical approach (see [Las]) based on the choice of $\mathcal{H}=D=(-\Delta)^{1 / 2}$ as a Hamiltonian of the process.

We shall observe some new interesting phenomena. On one hand, the contraction of some Sobolev norms of the solutions to (1) is manifested only for positive time $t>0$, and therefore we have a similarity to a diffusion type process.

The Cauchy problem for (11) has different conserved (or bounded) quantities that can be compared with the classical NLS with self interaction term

$$
\begin{cases}i \partial_{t} u-(-\Delta)^{1 / 2} u=-|u|^{p-1} u, & t \in \mathbb{R}, \quad x \in \mathbb{R}^{n}, \\ u(0)=u_{0}, & x \in \mathbb{R}^{n},\end{cases}
$$

Indeed, natural Sobolev norm that can be controlled for (3) is $H^{1 / 2}\left(\mathbb{R}^{n}\right)$, while (1) enables one to control $H^{1}\left(\mathbb{R}^{n}\right)$ norm but only in the future time instants $t>0$.

To state our main result, we turn to the introduction of the notations used below. For a Banach space $X$ and $1 \leq p \leq \infty$ let $L^{p}\left(\mathbb{R}^{n} ; X\right)$ be a $X$ valued Lebesgue space of $p$-th power. We abbreviate $L^{p}\left(\mathbb{R}^{n} ; \mathbb{C}\right)$ as $L^{p}\left(\mathbb{R}^{n}\right)$. For $f, g \in L^{2}\left(\mathbb{R}^{n}\right)$, we define a inner product as

$$
\langle f, g\rangle_{L^{2}\left(\mathbb{R}^{n}\right)}=\int_{\mathbb{R}^{n}} f(x) \bar{g}(x) d x .
$$

For $s \in \mathbb{R}$, let $H^{s}\left(\mathbb{R}^{n}\right)$ be the usual inhomogeneous Sobolev space defined as $H^{s}\left(\mathbb{R}^{n}\right)=(1-\Delta)^{-s / 2} L^{2}\left(\mathbb{R}^{n}\right)$. Let $\dot{H}^{s}\left(\mathbb{R}^{n}\right)$ be the usual homogeneous Sobolev space defined as $\dot{H}^{s}\left(\mathbb{R}^{n}\right)=(-\Delta)^{-s / 2} L^{2}\left(\mathbb{R}^{n}\right)$. For $f, g: A \rightarrow[0, \infty)$ with a set $A, f \lesssim g$ means there exists $C>0$ for any $a \in A$ such that $f(a) \leq C g(a)$. For Banach spaces $X, Y, Y \hookrightarrow X$ means $Y \subset X$ with continuous embedding. Moreover, we say a Cauchy problem is locally wellposed in $X$, if for any $X$-valued initial data, there exists $T>0$ and a Banach space $Y \hookrightarrow C([0, T] ; X)$ such that there is a unique solution to the Cauchy problem in $Y$ and $\left\|u_{n}-u\right\|_{Y} \rightarrow 0$ as $\left\|u_{0, n}-u_{0}\right\|_{X} \rightarrow 0$, where $u_{n}$ and $u$ are solutions for the Cauchy problem for initial data $u_{0}$ and $u_{0, n}$, respectively. We also say a Cauchy problem is globally well-posed in $X$ if the Cauchy problem is locally well-posed for any $T>0$. Moreover, we also say a Cauchy problem is globally well-posed in $X$ with sufficiently small data, if we have the property above for sufficiently small $X$-valued data.

(11) with is invariant under the scale transformation

$$
u_{\lambda}(t, x)=\lambda^{1 /(p-1)} u(\lambda t, \lambda x)
$$

with $\lambda>0$. Then

$$
\left\|u_{0, \sigma}\right\|_{\dot{H}^{s}\left(\mathbb{R}^{n}\right)}=\sigma^{1 /(p-1)+s-n / 2}\left\|u_{0}\right\|_{\dot{H}^{s}\left(\mathbb{R}^{n}\right)}
$$

and with

$$
s=s_{n, p}:=n / 2-1 /(p-1)<n / 2,
$$


$\dot{H}^{s}$ norm of initial data is also invariant. $s_{n, p}$ is called scale critical exponent. We also call $p_{n, s}=1+2 /(n-2 s)$ the $H^{s}\left(\mathbb{R}^{n}\right)$ scaling critical power. For any $s$, in the scaling subcritical case where $p<p_{n, s}$, (11) is expected to have local solution for any $H^{s}\left(\mathbb{R}^{n}\right)$ initial data on the analogy of scaling invariant Schrödinger equation. For instance, we refer the reader [C, CW1, CW2, II, IW]. However, with power type nonlinearity without gauge invariance, semirelativistic equations may not be locally well-posed even in scaling subcritical case, see $[\mathrm{F}]$.

In this paper, we show the following global well-posedness for the Cauchy problem (1):

Proposition 1.1. Let $n=1$. For $p>1$ the Cauchy problem (11) is globally well-posed in $H^{1}\left(\mathbb{R}^{1}\right)$. Moreover, for $p=3$, the Cauchy problem (11) is globally well-posed in $H^{s}\left(\mathbb{R}^{1}\right)$ with $1<s \leq 2$.

Proposition 1.2. Let $n=2$. For $p>1$ and $3 / 4<s<p$, the Cauchy problem (1) is locally well-posed in $H^{s}\left(\mathbb{R}^{2}\right)$. Moreover, for $p>1$, the Cauchy problem (11) is globally well-posed in $H^{1}\left(\mathbb{R}^{2}\right)$. For $p=3$, the Cauchy problem (11) is globally well-posed in $H^{s}\left(\mathbb{R}^{2}\right)$ with $1<s \leq 2$.

Proposition 1.3. Let $n \geq 3$ and $u_{0}$ be radial. For $1<p<p_{n, 1}=1+\frac{2}{n-2}$, the Cauchy problem (1) is globally well-posed in $H_{\mathrm{rad}}^{1}\left(\mathbb{R}^{3}\right)$.

Proposition 1.4. Let $n=3$ and $u_{0}$ be radial. For $p=p_{3,1}=3$, the Cauchy problem of (10) is globally well-posed in $H_{\mathrm{rad}}^{1}\left(\mathbb{R}^{3}\right)$ with sufficiently small $H_{\text {rad }}^{1}\left(\mathbb{R}^{3}\right)$ data.

For three dimensional case $p=3$ is a critical value in view of the result in [I]. However, the result in [I] treats nongauge invariant nonlinearities having constant sign, for which the test function method works. The question of the existence of local and global solutions for $n \geq 3$ and $p \geq 1+2 /(n-2)$ seems still open.

This paper is organized as follows: In section 2, we collect a priori estimates for (1). In section 3, we prove Propositions 1.1, 1.2, 1.3, and 1.4. In one dimensional case, local well-posedness follows from a standard contraction argument. In the case where $n=2$, local well-posedness follows from the Strichartz estimate derived by Nakamura and one of the authors in [NO]. However, with this Strichartz estimate, we may control solutions uniformly only in the $H^{s}\left(\mathbb{R}^{n}\right)$ setting with $s>(n+1) / 4$. We remark that it seems difficult to obtain the local well-posedness if $s \leq(n+1) / 4$ by a simple application of an improved Strichartz estimate for radial solutions derived by Guo and Wang in [GW]. In the case where $n \geq 3$, a weighted Sobolev space derived by Bellazzini, Visciglia, and one of the author in BGN, and uniform controls derived by Sickel and Skrzypczak in [SS] (see also [CO]) play an critical role to prove local well-posedness. Moreover, in the 3 dimensional scaling critical case where $p=3$, we obtain a uniform control of solutions by transforming (1) into the corresponding 1 dimensional problem. 


\section{A PRIORI EStimates}

Here we collect some a priori estimates. The Cauchy problem (1) is rewritten as the following integral equation:

$$
u(t)=U(t) u_{0}-\int_{0}^{t} U\left(t-t^{\prime}\right)\left|u\left(t^{\prime}\right)\right|^{p-1} u\left(t^{\prime}\right) d t^{\prime},
$$

where $U(t)=e^{-i t D}$ and $D=(-\Delta)^{1 / 2}$.

Proposition 2.1. Let $n \in \mathbb{N}$ and $p>1$. Let $u_{0} \in L^{2}\left(\mathbb{R}^{n}\right)$ and $T>0$. Let $u \in L^{\infty}\left(0, T ; L^{2}\left(\mathbb{R}^{n}\right)\right) \cap L^{p}\left(0, T ; L^{2 p}\left(\mathbb{R}^{n}\right)\right)$ be a solution to the integral equation (44) for the initial data $u_{0}$. Then, for any $t_{1}, t_{2}$ with $0<t_{1}<t_{2}<T$,

$$
\left\|u\left(t_{2}\right)\right\|_{L^{2}\left(\mathbb{R}^{n}\right)}^{2}+2\|u\|_{L^{p+1}\left(t_{1}, t_{2} ; L^{p+1}\left(\mathbb{R}^{n}\right)\right)}^{p+1}=\left\|u\left(t_{1}\right)\right\|_{L^{2}\left(\mathbb{R}^{n}\right)}^{2} .
$$

Proof. A formal computation yields immediately the proposition. However, actual proof requires some regularization procedure to justify the formal calculation. Here we give a direct proof based on the integral equation on the basis of the method in $\mathrm{O}$.

$$
\begin{aligned}
& \left\langle u\left(t_{2}\right), u\left(t_{2}\right)\right\rangle_{L^{2}\left(\mathbb{R}^{n}\right)} \\
& =\left\langle U\left(t_{2}-t_{1}\right) u\left(t_{1}\right)-\int_{t_{1}}^{t_{2}} U\left(t_{2}-t\right)|u(t)|^{p-1} u(t) d t, u\left(t_{2}\right)\right\rangle_{L^{2}\left(\mathbb{R}^{n}\right)} \\
& =\left\|u\left(t_{1}\right)\right\|_{L^{2}\left(\mathbb{R}^{n}\right)}^{2}-2 \operatorname{Re}\left\langle U\left(t_{2}-t_{1}\right) u\left(t_{1}\right), \int_{t_{1}}^{t_{2}} U\left(t_{2}-t\right)|u(t)|^{p-1} u(t) d t\right\rangle_{L^{2}\left(\mathbb{R}^{n}\right)} \\
& +\left\langle\int_{t_{1}}^{t_{2}} U\left(t_{2}-t\right)|u(t)|^{p-1} u(t) d t, \int_{t_{1}}^{t_{2}} U\left(t_{2}-t^{\prime}\right)\left|u\left(t^{\prime}\right)\right|^{p-1} u\left(t^{\prime}\right) d t^{\prime}\right\rangle_{L^{2}\left(\mathbb{R}^{n}\right)} \\
& =\left\|u\left(t_{1}\right)\right\|_{L^{2}\left(\mathbb{R}^{n}\right)}^{2}-2 \operatorname{Re}\left\langle U\left(t_{2}-t_{1}\right) u\left(t_{1}\right), \int_{t_{1}}^{t_{2}} U\left(t_{2}-t\right)|u(t)|^{p-1} u(t) d t\right\rangle_{L^{2}\left(\mathbb{R}^{n}\right)} \\
& +2 \operatorname{Re} \int_{t_{1}}^{t_{2}}\left\langle|u(t)|^{p-1} u(t), \int_{t_{1}}^{t} U\left(t-t^{\prime}\right)\left|u\left(t^{\prime}\right)\right|^{p-1} u\left(t^{\prime}\right) d t^{\prime}\right\rangle_{L^{2}\left(\mathbb{R}^{n}\right)} d t \\
& =\left\|u\left(t_{1}\right)\right\|_{L^{2}\left(\mathbb{R}^{n}\right)}^{2}-2 \operatorname{Re}\left\langle U\left(t_{2}-t_{1}\right) u\left(t_{1}\right), \int_{t_{1}}^{t_{2}} U\left(t_{2}-t\right)|u(t)|^{p-1} u(t) d t\right\rangle_{L^{2}\left(\mathbb{R}^{n}\right)} \\
& +2 \operatorname{Re} \int_{t_{1}}^{t_{2}}\left\langle|u(t)|^{p-1} u(t), U\left(t-t_{1}\right) u\left(t_{1}\right)-u(t)\right\rangle_{L^{2}\left(\mathbb{R}^{n}\right)} d t \\
& =\left\|u\left(t_{1}\right)\right\|_{L^{2}\left(\mathbb{R}^{n}\right)}^{2}-2\|u\|_{L^{p+1}\left(t_{1}, t_{2} ; L^{p+1}\left(\mathbb{R}^{n}\right)\right)}^{p+1}
\end{aligned}
$$

Proposition 2.2. Let $n \in \mathbb{N}$ and $p>1$. Let $u_{0} \in H^{1}\left(\mathbb{R}^{n}\right)$ and $T>0$. Let $u \in L^{\infty}\left(0, T ; H^{1}\left(\mathbb{R}^{n}\right)\right) \cap L^{p-1}\left(0, T ; L^{\infty}\left(\mathbb{R}^{n}\right)\right)$ be a solution to the integral equation (4) for the initial data $u_{0}$. Then, for any $t_{1}, t_{2}$ with $0 \leq t_{1}<t_{2} \leq T$,

$$
\begin{aligned}
& \left\|\nabla u\left(t_{2}\right)\right\|_{L^{2}\left(\mathbb{R}^{n}\right)}^{2}+2\left\|\left.|| u\right|^{\frac{p-1}{2}} \nabla u\right\|_{L^{2}\left(t_{1}, t_{2} ; L^{2}\left(\mathbb{R}^{n}\right)\right)}^{2} \\
& +\frac{p-1}{2}\left\||u|^{\frac{p-3}{2}} \nabla|u|^{2}\right\|_{L^{2}\left(t_{1}, t_{2} ; L^{2}\left(\mathbb{R}^{n}\right)\right)}^{2} \\
& =\left\|\nabla u\left(t_{1}\right)\right\|_{L^{2}\left(\mathbb{R}^{n}\right)}^{2} .
\end{aligned}
$$


Proof. Since $|u|^{p-1} u \in L^{1}\left(0, T ; H^{1}\left(\mathbb{R}^{n}\right)\right)$,

$$
\begin{aligned}
& \left\|\nabla u\left(t_{2}\right)\right\|_{L^{2}\left(\mathbb{R}^{n}\right)}^{2} \\
& =\left\|\nabla u\left(t_{1}\right)\right\|_{L^{2}\left(\mathbb{R}^{n}\right)}^{2}-2 \operatorname{Re} \int_{t_{1}}^{t_{2}}\left\langle\nabla\left(|u(t)|^{p-1} u(t)\right), \nabla u(t)\right\rangle_{L^{2}\left(\mathbb{R}^{n}\right)} d t \\
& =\left\|\nabla u\left(t_{1}\right)\right\|_{L^{2}\left(\mathbb{R}^{n}\right)}^{2}-2 \operatorname{Re} \int_{t_{1}}^{t_{2}}\left\langle\nabla|u(t)|^{p-1}, \overline{u(t)} \nabla u(t)\right\rangle_{L^{2}\left(\mathbb{R}^{n}\right)} d t \\
& -2 \int_{t_{1}}^{t_{2}}\left\langle|u(t)|^{p-1} \nabla u(t), \nabla u(t)\right\rangle_{L^{2}\left(\mathbb{R}^{n}\right)} d t \\
& =\left\|\nabla u\left(t_{1}\right)\right\|_{L^{2}\left(\mathbb{R}^{n}\right)}^{2}-\frac{p-1}{2} \int_{t_{1}}^{t_{2}}\left\langle|u(t)|^{p-3} \nabla|u(t)|^{2}, \nabla|u(t)|^{2}\right\rangle_{L^{2}\left(\mathbb{R}^{n}\right)} d t \\
& -2 \int_{t_{1}}^{t_{2}}\left\langle|u(t)|^{p-1} \nabla u(t), \nabla u(t)\right\rangle_{L^{2}\left(\mathbb{R}^{n}\right)} d t .
\end{aligned}
$$

Proposition 2.3. Let $n=1,2, p>1, n / 2<s<\min (2, p)$, and $T>0$. Let $u_{0} \in H^{s}\left(\mathbb{R}^{n}\right)$ and $u \in L^{\infty}\left(0, T ; H^{s}\left(\mathbb{R}^{n}\right)\right) \cap L^{2}\left(0, T ; L^{\infty}\left(\mathbb{R}^{n}\right)\right)$ be a solution to (41) for the initial data $u_{0}$. Then for any $t_{1}, t_{2}$ with $0<t_{1}<t_{2}<T$,

$$
\left\|u\left(t_{2}\right)\right\|_{\dot{H}^{s}\left(\mathbb{R}^{n}\right)}^{2} \leq\left\|u\left(t_{1}\right)\right\|_{\dot{H}^{s}\left(\mathbb{R}^{n}\right)}^{2}+C \int_{t_{1}}^{t_{2}}\|u(t)\|_{L^{\infty}\left(\mathbb{R}^{n}\right)}^{p-1}\|u(t)\|_{\dot{H}^{s}\left(\mathbb{R}^{n}\right)}^{2} d t .
$$

Proof.

$$
\begin{aligned}
& \left\|u\left(t_{2}\right)\right\|_{\dot{H}^{s}\left(\mathbb{R}^{n}\right)}^{2} \\
& =\left\|u\left(t_{1}\right)\right\|_{\dot{H}^{s}\left(\mathbb{R}^{n}\right)}^{2}-2 \operatorname{Re} \int_{t_{1}}^{t_{2}}\left\langle D^{s}\left(|u(t)|^{p-1} u(t)\right), D^{s} u(t)\right\rangle_{L^{2}\left(\mathbb{R}^{n}\right)} d t \\
& \leq\left\|u\left(t_{1}\right)\right\|_{\dot{H}^{s}\left(\mathbb{R}^{n}\right)}^{2}+2 \int_{t_{1}}^{t_{2}}\left\|D^{s}\left(|u(t)|^{p-1} u(t)\right)\right\|_{L^{2}\left(\mathbb{R}^{n}\right)}\|u(t)\|_{\dot{H}^{s}\left(\mathbb{R}^{n}\right)} d t \\
& \leq\left\|u\left(t_{1}\right)\right\|_{\dot{H}^{s}\left(\mathbb{R}^{n}\right)}^{2}+C \int_{t_{1}}^{t_{2}}\|u(t)\|_{L^{\infty}\left(\mathbb{R}^{n}\right)}^{p-1}\|u(t)\|_{\dot{H}^{s}\left(\mathbb{R}^{n}\right)}^{2} d t,
\end{aligned}
$$

where we use the nonlinear estimate

$$
\left\||f|^{p-1} f\right\|_{\dot{H}^{s}\left(\mathbb{R}^{n}\right)} \lesssim\|f\|_{L^{\infty}\left(\mathbb{R}^{n}\right)}^{p-1}\|f\|_{\dot{H}^{s}\left(\mathbb{R}^{n}\right)}
$$

(see GOV, Lemma 3.4]).

Proposition 2.4. Let $1 \leq n \leq 3, u_{0} \in H^{2}\left(\mathbb{R}^{n}\right)$ and $T>0$. Let $u \in$ $C\left((0, T) ; H^{2}\left(\mathbb{R}^{n}\right) \cap L^{\infty}\left(\mathbb{R}^{n}\right)\right)$ be a solution to the integral equation (4) for the initial data $u_{0}$. Then, for any $t_{1}, t_{2}$ with $0<t_{1}<t_{2}<T$,

$$
\begin{aligned}
& \left\|u\left(t_{2}\right)\right\|_{\dot{H}^{2}\left(\mathbb{R}^{n}\right)}^{2}+2 \sum_{j, k=1}^{n} \int_{t_{1}}^{t_{2}}\left\|u(t) \partial_{j} \partial_{k} u(t)\right\|_{L^{2}\left(\mathbb{R}^{n}\right)}^{2} d t \\
& \quad \leq\left\|u\left(t_{1}\right)\right\|_{\dot{H}^{2}\left(\mathbb{R}^{n}\right)}^{2}+2 n^{2}(n+1) \int_{t_{1}}^{t_{2}}\|u(t)\|_{\dot{H}^{1}\left(\mathbb{R}^{n}\right)}^{4-n}\|u(t)\|_{\dot{H}^{2}\left(\mathbb{R}^{n}\right)}^{n} d t .
\end{aligned}
$$


Proof. Since $|u|^{2} u \in C\left((0, T) ; H^{2}\left(\mathbb{R}^{n}\right)\right)$, the following calculation is justified by the Plancherel identity:

$$
\begin{aligned}
& \left\|u\left(t_{2}\right)\right\|_{\dot{H}^{2}\left(\mathbb{R}^{n}\right)}^{2} \\
& =\left\|u\left(t_{1}\right)\right\|_{\dot{H}^{2}\left(\mathbb{R}^{n}\right)}^{2}-2 \operatorname{Re} \int_{t_{1}}^{t_{2}}\left\langle\Delta|u(t)|^{2} u(t), \Delta u(t)\right\rangle_{L^{2}\left(\mathbb{R}^{n}\right)} d t \\
& =\left\|u\left(t_{1}\right)\right\|_{\dot{H}^{2}\left(\mathbb{R}^{n}\right)}^{2}-2 \operatorname{Re} \sum_{j, k=1}^{n} \int_{t_{1}}^{t_{2}}\left\langle|u(t)|^{2} \partial_{j} \partial_{k} u(t), \partial_{j} \partial_{k} u(t)\right\rangle_{L^{2}\left(\mathbb{R}^{n}\right)} d t \\
& -4 \operatorname{Re} \sum_{j, k=1}^{n} \int_{t_{1}}^{t_{2}}\left\langle\partial_{k} u(t) \partial_{j}|u(t)|^{2}, \partial_{j} \partial_{k} u(t)\right\rangle_{L^{2}\left(\mathbb{R}^{n}\right)} d t \\
& -2 \operatorname{Re} \sum_{j, k=1}^{n} \int_{t_{1}}^{t_{2}}\left\langle\partial_{j} \partial_{k}|u(t)|^{2}, \overline{u(t)} \partial_{j} \partial_{k} u(t)\right\rangle_{L^{2}\left(\mathbb{R}^{n}\right)} d t \\
& =\left\|u\left(t_{1}\right)\right\|_{\dot{H}^{2}\left(\mathbb{R}^{n}\right)}^{2}-2 \sum_{j, k=1}^{n} \int_{t_{1}}^{t_{2}}\left\|u(t) \partial_{j} \partial_{k} u(t)\right\|_{L^{2}\left(\mathbb{R}^{n}\right)}^{2} d t \\
& +2 \sum_{j, k=1}^{n} \int_{t_{1}}^{t_{2}}\left\langle\partial_{j}^{2}|u(t)|^{2},\left|\partial_{k} u(t)\right|^{2}\right\rangle_{L^{2}\left(\mathbb{R}^{n}\right)} d t \\
& -\sum_{j, k=1}^{n} \int_{t_{1}}^{t_{2}}\left\langle\partial_{j} \partial_{k}|u(t)|^{2}, \partial_{j} \partial_{k}|u(t)|^{2}-2 \operatorname{Re}\left(\overline{\partial_{j} u(t)} \partial_{k} u(t)\right)\right\rangle_{L^{2}\left(\mathbb{R}^{n}\right)} d t .
\end{aligned}
$$

By the Young inequality,

$$
\begin{aligned}
& \sum_{j, k=1}^{n}\left\langle\partial_{j}^{2}|u(t)|^{2},\left|\partial_{k} u(t)\right|^{2}\right\rangle_{L^{2}\left(\mathbb{R}^{n}\right)} \\
& \leq \sum_{j, k=1}^{n}\left\|\partial_{j}^{2}|u(t)|^{2}\right\|_{L^{2}\left(\mathbb{R}^{n}\right)}\left\|\partial_{k} u(t)\right\|_{L^{4}\left(\mathbb{R}^{n}\right)}^{2} \\
& \leq \frac{1}{4} \sum_{j=1}^{n}\left\|\partial_{j}^{2}|u(t)|^{2}\right\|_{L^{2}\left(\mathbb{R}^{n}\right)}^{2}+n^{2} \sum_{k=1}^{n}\left\|\partial_{k} u(t)\right\|_{L^{4}\left(\mathbb{R}^{n}\right)}^{4} \\
& \leq \frac{1}{4} \sum_{j, k=1}^{n}\left\|\partial_{j} \partial_{k}|u(t)|^{2}\right\|_{L^{2}\left(\mathbb{R}^{n}\right)}^{2}+n^{2} \sum_{k=1}^{n}\left\|\partial_{k} u(t)\right\|_{L^{4}\left(\mathbb{R}^{n}\right)}^{4}
\end{aligned}
$$

Similarly,

$$
\begin{aligned}
& \sum_{j, k=1}^{n}\left\langle\partial_{j} \partial_{k}|u(t)|^{2}, \operatorname{Re}\left(\overline{\partial_{j} u(t)} \partial_{k} u(t)\right)\right\rangle_{L^{2}\left(\mathbb{R}^{n}\right)} \\
& \leq \frac{1}{4} \sum_{j, k=1}^{n}\left\|\partial_{j} \partial_{k}|u(t)|^{2}\right\|_{L^{2}\left(\mathbb{R}^{n}\right)}^{2}+\sum_{j, k=1}^{n}\left\|\partial_{j} u(t)\right\|_{L^{4}\left(\mathbb{R}^{n}\right)}^{2}\left\|\partial_{k} u(t)\right\|_{L^{4}\left(\mathbb{R}^{n}\right)}^{2} .
\end{aligned}
$$


Therefore, by the Sobolev inequality,

$$
\begin{aligned}
& \left\|u\left(t_{2}\right)\right\|_{\dot{H}^{2}\left(\mathbb{R}^{n}\right)}^{2} \\
& \leq\left\|u\left(t_{1}\right)\right\|_{\dot{H}^{2}\left(\mathbb{R}^{n}\right)}^{2}-2 \sum_{j, k=1}^{n} \int_{t_{1}}^{t_{2}}\left\|u(t) \partial_{j} \partial_{k} u(t)\right\|_{L^{2}\left(\mathbb{R}^{n}\right)}^{2} d t \\
& +2 n^{2} \sum_{k=1}^{n} \int_{t_{1}}^{t_{2}}\left\|\partial_{k} u(t)\right\|_{L^{4}\left(\mathbb{R}^{n}\right)}^{4} d t+2 \sum_{j, k=1}^{n} \int_{t_{1}}^{t_{2}}\left\|\partial_{j} u(t)\right\|_{L^{4}\left(\mathbb{R}^{n}\right)}^{2}\left\|\partial_{k} u(t)\right\|_{L^{4}\left(\mathbb{R}^{n}\right)}^{2} d t \\
& \leq\left\|u\left(t_{1}\right)\right\|_{\dot{H}^{2}\left(\mathbb{R}^{n}\right)}^{2}-2 \sum_{j, k=1}^{n} \int_{t_{1}}^{t_{2}}\left\|u(t) \partial_{j} \partial_{k} u(t)\right\|_{L^{2}\left(\mathbb{R}^{n}\right)}^{2} d t \\
& +2 n^{2}(n+1) \int_{t_{1}}^{t_{2}}\|u(t)\|_{\dot{H}^{1}\left(\mathbb{R}^{n}\right)}^{4-n}\|u(t)\|_{\dot{H}^{2}\left(\mathbb{R}^{n}\right)}^{n} d t .
\end{aligned}
$$

\section{Proof of the Propositions}

3.1. 1 dimensional case. Since $L^{\infty}(\mathbb{R}) \hookrightarrow H^{1}(\mathbb{R}) \hookrightarrow H^{2}(\mathbb{R})$, Proposition 1.1 is obtained by a standard contraction argument with the Sobolev inequality and a priori estimates Propositions 2.1, 2.2, 2.3, and 2.4.

3.2. $\mathbf{2}$ dimensional case. In two dimensional case, the local well-posedness may be obtained by the following Strichartz estimates:

Lemma 3.1 ([NO, Lemma 2.1], GV, Remark 3.2]). Let $n=1,2,3$. Let

$$
\alpha(r)=\frac{1}{2}-\frac{1}{r}, \quad \lambda=\frac{n+1}{2}, \quad \sigma=n-1 .
$$

Let $\left(q_{1}, r_{1}\right)$ and $\left(q_{2}, r_{2}\right)$ satisfy

$$
\frac{1}{r_{j}}=\frac{1}{2}-\frac{2}{\sigma} \frac{1}{q_{j}}
$$

and $2 \leq r_{j} \leq \infty$ if $n=1,2$ and $2 \leq r_{j}<\infty$ if $n=3$ for $j=1,2$. Then for $s \in \mathbb{R}$,

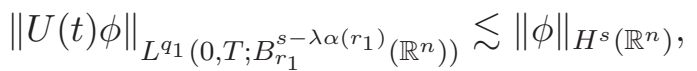

$$
\begin{aligned}
& \left\|\int_{0}^{t} U\left(t-t^{\prime}\right) h\left(t^{\prime}\right) d t^{\prime}\right\|_{L^{q_{1}\left(0, T ; B_{r_{1}}^{s-\lambda \alpha\left(r_{1}\right)}\left(\mathbb{R}^{n}\right)\right)}} \lesssim\|h\|_{L^{q_{2}^{\prime}\left(0, T ; B_{r_{2}^{\prime}}^{s-\lambda \alpha\left(r_{2}^{\prime}\right)}\left(\mathbb{R}^{n}\right)\right)}},
\end{aligned}
$$

where $B_{p, q}^{s}\left(\mathbb{R}^{n}\right)$ is the usual inhomogeneous Besov space.

Lemma 3.2. Let $r>2$, and $T>0$. If $q \geq 4$ and

$$
s>\frac{3}{4}+\frac{1}{2 r},
$$

then $L^{q}\left(0, T ; B_{r}^{s-3 / 2 \cdot \alpha(r)}\left(\mathbb{R}^{2}\right)\right) \hookrightarrow L^{4}\left(0, T ; L^{\infty}\left(\mathbb{R}^{2}\right)\right)$.

Proof. Since

$$
\begin{aligned}
& s-\frac{3}{2} \alpha(r)-\frac{2}{r}>0 \Longleftrightarrow s>\frac{3}{4}+\frac{1}{2 r}, \\
& L^{q}\left(0, T ; B_{r}^{s-3 / 2 \cdot \alpha(r)}\left(\mathbb{R}^{2}\right)\right) \hookrightarrow L^{4}\left(0, T ; L^{\infty}\left(\mathbb{R}^{2}\right)\right) .
\end{aligned}
$$


Proof of Proposition 1.2.

Local well-posedness Let $\left(q_{1}, r_{1}\right)$ satisfy the condition of Lemma 3.2, (7), and $q_{1}>p-1$. Let $q_{2}=\infty$ and $r_{2}=2$. Let $X^{s}(0, T)=L^{\infty}\left(0, T ; H^{s}\left(\mathbb{R}^{2}\right)\right) \cap$ $L^{q_{1}}\left(0, T ; B_{r_{1}}^{s-3 / 2 \cdot \alpha\left(r_{1}\right)}\left(\mathbb{R}^{2}\right)\right)$. Let

$$
\Phi(u)(t)=U(t) u_{0}-\int_{0}^{t} U\left(t-t^{\prime}\right)\left|u\left(t^{\prime}\right)\right|^{p-1} u\left(t^{\prime}\right) d t^{\prime} .
$$

Then, for $0<T<1$,

$$
\begin{aligned}
\|\Phi(u)\|_{X^{s}(0, T)} & \leq\left\|u_{0}\right\|_{H^{s}\left(\mathbb{R}^{2}\right)}+C\left\|\left.|| u\right|^{p-1} u\right\|_{L^{1}\left(0, T ; H^{s}\left(\mathbb{R}^{2}\right)\right)} \\
& \leq\left\|u_{0}\right\|_{H^{s}\left(\mathbb{R}^{2}\right)}+C T^{1-(p-1) / q_{1}}\|u\|_{X^{s}(0, T)}^{p}
\end{aligned}
$$

and

$$
\begin{aligned}
& \|\Phi(u)-\Phi(v)\|_{X^{s}(0, T)} \\
& \leq\left\|u_{0}-v_{0}\right\|_{H^{s}(\mathbb{R})}+C\left\||u|^{p-1} u-|v|^{p-1} v\right\|_{L^{1}\left(0, T ; H^{s}\left(\mathbb{R}^{2}\right)\right)} \\
& \leq\left\|u_{0}-v_{0}\right\|_{H^{s}(\mathbb{R})} \\
& +C T^{1-(p-1) / q_{1}}\left(\|u\|_{X^{s}(0, T)}+\|v\|_{X^{s}(0, T)}\right)^{p-1}\|u-v\|_{X^{s}(0, T)} \\
& +C T^{1-(p-1) / q_{1}}\left(\|u\|_{X^{s}(0, T)}+\|v\|_{X^{s}(0, T)}\right)^{\max (1, p-1)}\|u-v\|_{X^{s}(0, T)}^{\min (1, p-1)}
\end{aligned}
$$

This means if $T<T_{0}:=\left(2^{1+\max (1, p-1)} C\left(\left\|u_{0}\right\|_{H^{s}\left(\mathbb{R}^{2}\right)}^{p-1}+\left\|u_{0}\right\|_{H^{s}\left(\mathbb{R}^{2}\right)}^{\max (1, p-1)}\right)\right)^{-q_{1} /\left(q_{1}-p+1\right)}$, then $\Phi$ is a map from

$$
B_{X^{s}(0, T)}\left(2\left\|u_{0}\right\|_{H^{s}\left(\mathbb{R}^{2}\right)}\right):=\left\{f \in X^{s}(0, T) \mid\|f\|_{X^{s}(0, T)} \leq 2\left\|u_{0}\right\|_{H^{s}\left(\mathbb{R}^{2}\right)}\right\} .
$$

to itself. Moreover, if $p \geq 2, \Phi$ is a contraction map in $X^{s}(0, T)$. If $p<2$, since for $z_{1}, z_{0} \in \mathbb{C}$ with $\left|z_{1}\right|>\left|z_{0}\right|$,

$$
\begin{aligned}
& \left.|| z_{1}\right|^{p-1} z_{1}-\left|z_{0}\right|^{p-1} z_{0} \mid \\
& \leq\left|z_{1}\right|^{p-1}\left|z_{1}-z_{0}\right|+\frac{1}{p-1} \int_{0}^{1}\left(\theta\left|z_{1}\right|+(1-\theta)\left|z_{0}\right|\right)^{p-2}\left|z_{0}\right|\left|z_{1}-z_{0}\right| \\
& \leq\left|z_{1}\right|^{p-1}\left|z_{1}-z_{0}\right|+\frac{1}{p-1}\left|z_{0}\right|^{p-1}\left|z_{1}-z_{0}\right|
\end{aligned}
$$

then

(9)

$$
\begin{aligned}
& \|\Phi(u)-\Phi(v)\|_{L^{\infty}\left(0, T ; L^{2}\left(\mathbb{R}^{2}\right)\right)} \\
& \leq\left\|u_{0}-v_{0}\right\|_{L^{2}\left(\mathbb{R}^{2}\right)}+C\left\|\left.|| u\right|^{p-1} u-|v|^{p-1} v\right\|_{L^{1}\left(0, T ; L^{2}\left(\mathbb{R}^{2}\right)\right)} \\
& \leq\left\|u_{0}-v_{0}\right\|_{L^{2}\left(\mathbb{R}^{2}\right)} \\
& +C T^{1-(p-1) / q_{1}}\left(\|u\|_{X^{s}(0, T)}+\|v\|_{X^{s}(0, T)}\right)^{p-1}\|u-v\|_{L^{\infty}\left(0, T ; L^{2}\left(\mathbb{R}^{2}\right)\right)}
\end{aligned}
$$

and therefore $\Phi$ is a contraction map in $L^{\infty}\left(0, T ; L^{2}\left(\mathbb{R}^{2}\right)\right)$. Let $u_{1} \in B_{X^{s}(0, T)}\left(2\left\|u_{0}\right\|_{H^{s}\left(\mathbb{R}^{2}\right)}\right)$ and $u_{k}=\Phi\left(u_{k-1}\right)$ for $k \geq 2$. Then $\left(u_{k}\right)_{k=1}^{\infty} \subset B_{X^{s}(0, T)}\left(2\left\|u_{0}\right\|_{H^{s}\left(\mathbb{R}^{2}\right)}\right)$ is a Cauchy sequence in $L^{\infty}\left(0, T ; L^{2}\left(\mathbb{R}^{2}\right)\right)$. Let $u^{*}$ be the limit of $\left(u_{k}\right)_{k=1}^{\infty}$ in $L^{\infty}\left(0, T ; L^{2}\left(\mathbb{R}^{2}\right)\right)$. Since

$$
\left\||f|^{p-1} g\right\|_{L^{2 / p}\left(\mathbb{R}^{n}\right)} \leq\|f\|_{L^{2}\left(\mathbb{R}^{n}\right)}^{p-1}\|g\|_{L^{2}\left(\mathbb{R}^{n}\right)}
$$

for any $n \in \mathbb{N}$ and $f, g \in L^{2}\left(\mathbb{R}^{n}\right), \Phi\left(u_{k}\right) \rightarrow \Phi\left(u^{*}\right)$ in $L^{\infty}\left(0, T ; L^{2}\left(\mathbb{R}^{2}\right)\right)$.

Therefore $u^{*}$ is a solution of (4). Moreover, since $X^{s}(0, T) \hookrightarrow L^{\infty}\left(0, T ; H^{s}\left(\mathbb{R}^{2}\right)\right)$, 
$u^{*}$ is also in $L^{\infty}\left(0, T ; H^{s}\left(\mathbb{R}^{2}\right)\right)$. By (12),

$$
u^{*} \in L^{q_{1}}\left(0, T ; B_{r_{1}}^{s-\frac{3}{2} \alpha\left(r_{1}\right)}\left(\mathbb{R}^{2}\right)\right) .
$$

If $s>1$, by the Sobolev embedding, for some $0<\theta<1$,

$$
\|u-v\|_{L^{\infty}\left(0, T ; L^{\infty}\left(\mathbb{R}^{2}\right)\right)} \lesssim\|u-v\|_{L^{\infty}\left(0, T ; L^{2}\left(\mathbb{R}^{2}\right)\right)}^{\theta}\|u-v\|_{L^{\infty}\left(0, T ; H^{1}\left(\mathbb{R}^{2}\right)\right)}^{1-\theta}
$$

and therefore the solution map depends continuously on the initial data continuously in $H^{s}\left(\mathbb{R}^{2}\right)$. In the case where $s \leq 1$, the continuous dependence of $\Phi$ may be shown as follows. By (9), the solution map depends continuously on the initial data continuously in $L^{2}\left(\mathbb{R}^{2}\right)$. We define $s_{3}, s_{4}>0$ so that they satisfy the following: $\max \left(\frac{3}{4}+\frac{1}{2 r_{1}}, s_{4}-\frac{3}{4}(p-1)\right)<s_{3}<s_{4}<s$, $r_{3}=\frac{3}{2}\left(s_{3}-s_{4}+\frac{3}{4}\right)^{-1}$, and $q_{3}>p-1$, where $\left(q_{3}, r_{3}\right)$ satisfy (7). Then $B_{r_{1}}^{s_{3}-\frac{3}{2} \alpha\left(r_{1}\right)}\left(\mathbb{R}^{2}\right) \hookrightarrow L^{\infty}\left(\mathbb{R}^{2}\right), B_{r_{3}^{\prime}}^{s_{3}-\frac{3}{2} \alpha\left(r_{3}^{\prime}\right)}\left(\mathbb{R}^{2}\right)=B_{r_{3}^{\prime}}^{s_{4}}\left(\mathbb{R}^{2}\right), 2<r_{3}<\frac{3}{2} \frac{4}{3(2-p)}$, and $\frac{r_{3}(p-1)}{r_{3}-2}>1$. Moreover,

$$
\begin{aligned}
& \|\Phi(u)-\Phi(v)\|_{L^{q_{1}}\left(0, T ; B_{r_{1}}^{s_{3}}\left(\mathbb{R}^{2}\right)\right)} \\
& \leq\left\|u_{0}-v_{0}\right\|_{H^{s_{3}(\mathbb{R})}}+C\left\||u|^{p-1} u-|v|^{p-1} v\right\|_{L^{q_{3}^{\prime}}\left(0, T ; B_{r_{3}^{\prime}}^{s_{4}}\left(\mathbb{R}^{2}\right)\right)} .
\end{aligned}
$$

For $z_{j} \in \mathbb{C}$ with $j=1,2,3,4$, with $w_{1}=z_{2}-z_{1}$ and $w_{2}=z_{4}-z_{3}$,

$$
\begin{aligned}
& \left|z_{4}\right|^{p-1} z_{4}-\left|z_{3}\right|^{p-1} z_{3}-\left|z_{2}\right|^{p-1} z_{2}+\left|z_{1}\right|^{p-1} z_{1} \\
& =\frac{p+1}{2} \int_{0}^{1}\left|z_{3}+\theta w_{2}\right|^{p-1} d \theta w_{2}-\frac{p+1}{2} \int_{0}^{1}\left|z_{1}+\theta w_{1}\right|^{p-1} d \theta w_{1} \\
& +\frac{p-1}{2} \int_{0}^{1}\left|z_{3}+\theta w_{2}\right|^{p-3}\left(z_{3}+\theta w_{2}\right)^{2} d \theta \overline{w_{2}} \\
& -\frac{p-1}{2} \int_{0}^{1}\left|z_{1}+\theta w_{1}\right|^{p-3}\left(z_{1}+\theta w_{1}\right)^{2} d \theta \overline{w_{1}}
\end{aligned}
$$

Then

$$
\begin{aligned}
& \left|\int_{0}^{1}\right| z_{3}+\left.\theta w_{2}\right|^{p-1} d \theta w_{2}-\int_{0}^{1}\left|z_{1}+\theta w_{1}\right|^{p-1} d \theta w_{1} \mid \\
& \leq \int_{0}^{1}\left|z_{3}+\theta w_{2}\right|^{p-1} d \theta\left|w_{2}-w_{1}\right| \\
& +\int_{0}^{1}|| z_{3}+\left.\theta w_{2}\right|^{p-1}-\left|z_{1}+\theta w_{1}\right|^{p-1}|d \theta| w_{1} \mid \\
& \leq\left(\left|z_{3}\right|^{p-1}+\left|z_{4}\right|^{p-1}\right)\left|w_{2}-w_{1}\right|+\left|z_{3}-z_{1}\right|^{p-1}\left|w_{1}\right|+\frac{1}{p}\left|w_{1}\right|\left|w_{2}-w_{1}\right|^{p-1} \\
& \leq\left(\left|z_{3}\right|^{p-1}+\left|z_{4}\right|^{p-1}\right)\left|w_{2}-w_{1}\right|+\frac{p+1}{p}\left|w_{1}\right|\left|z_{3}-z_{1}\right|^{p-1}+\frac{1}{p}\left|w_{1}\right|\left|z_{4}-z_{2}\right|^{p-1} .
\end{aligned}
$$

Similarly

$$
\begin{aligned}
& \left|\int_{0}^{1}\right| z_{3}+\left.\theta w_{2}\right|^{p-3}\left(z_{3}+\theta w_{2}\right)^{2} d \theta \overline{w_{2}}-\int_{0}^{1}\left|z_{1}+\theta w_{1}\right|^{p-3}\left(z_{1}+\theta w_{1}\right)^{2} d \theta \overline{w_{1}} \mid \\
& \lesssim\left(\left|z_{3}\right|^{p-1}+\left|z_{4}\right|^{p-1}\right)\left|w_{2}-w_{1}\right|+\left|w_{1}\right|\left|z_{3}-z_{1}\right|^{p-1}+\left|w_{1}\right|\left|z_{4}-z_{2}\right|^{p-1}
\end{aligned}
$$


since

$$
\begin{aligned}
\left.|| z_{2}\right|^{p-3} z_{2}^{2}-\left|z_{1}\right|^{p-3} z_{1}^{2} \mid & \lesssim \int_{0}^{1}\left|z_{1}^{2}+\theta\left(z_{2}-z_{1}\right)\right|^{p-2} d \theta\left|z_{2}-z_{1}\right| \\
& =\int_{0}^{1}\left|z_{1}^{2}+\theta\left(z_{2}-z_{1}\right)\right|^{p-2} d \theta\left|z_{2}-z_{1}\right| \\
& \leq \int_{0}^{1}|| z_{1}|-\theta| z_{2}-z_{1}||^{p-2} d \theta\left|z_{2}-z_{1}\right| \\
& =\frac{1}{2-p}\left(\left|z_{1}\right|^{p-1}-|| z_{1}|-| z_{2}-z_{1}||^{p-1}\right) \\
& \leq\left|z_{2}-z_{1}\right|^{p-1} .
\end{aligned}
$$

Therefore,

$$
\begin{aligned}
& \||u(t, \cdot+h)|^{p-1} u(t, \cdot+h)-|v(t, \cdot+h)|^{p-1} v(t, \cdot+h) \\
& \quad-|u(t)|^{p-1} u(t)+|v(t)|^{p-1} v(t) \|_{L^{r_{3}^{\prime}\left(\mathbb{R}^{2}\right)}} \\
& =\||u(t, \cdot+h)|^{p-1} u(t, \cdot+h)-|u(t)|^{p-1} u(t) \\
& \quad-|v(t, \cdot+h)|^{p-1} v(t, \cdot+h)+|v(t)|^{p-1} v(t) \|_{L^{r_{3}^{\prime}}\left(\mathbb{R}^{2}\right)} \\
& \leq 4\|u(t)\|^{p-1}{ }_{L^{\frac{2 r_{3}(p-1)}{r_{3}-2}}}\left(\mathbb{R}^{2}\right) \\
& +\frac{2(p+2)}{p}\|v(t, \cdot+h)-v(t, \cdot+h)-u(t)+v(t)\|_{L^{2}\left(\mathbb{R}^{2}\right)}
\end{aligned}
$$

and this means

$$
\begin{aligned}
& \left\||u|^{p-1} u-|v|^{p-1} v\right\|_{L^{q_{3}^{\prime}}\left(0, T ; B_{r_{3}^{\prime}}^{s_{4}}\left(\mathbb{R}^{2}\right)\right)} \\
& \lesssim\|\| u\left\|_{L^{\frac{2 r_{3}(p-1)}{r_{3}-2}}\left(\mathbb{R}^{2}\right)}\right\| u-v\left\|_{H^{s_{4}\left(\mathbb{R}^{2}\right)}}+\right\| v\left\|_{H^{s_{4}\left(\mathbb{R}^{2}\right)}}\right\| u-v\left\|_{L^{\frac{2 r_{3}(p-1)}{r_{3}-2}}\left(\mathbb{R}^{2}\right)}\right\|_{L^{q_{3}^{\prime}(0, T)}} \\
& \leq\|\| u\left\|_{L^{\infty}\left(\mathbb{R}^{2}\right)}^{p-1-\frac{r_{3}-2}{r_{3}}}\right\| u\left\|_{L^{2}\left(\mathbb{R}^{2}\right)}^{\frac{r_{3}-2}{r_{3}}}\right\| u-v\left\|_{H^{s_{4}\left(\mathbb{R}^{2}\right)}}\right\|_{L^{q_{3}^{\prime}(0, T)}} \\
& +\|\| v\left\|_{H^{s_{4}\left(\mathbb{R}^{2}\right)}}\right\| u-v\left\|_{L^{\infty}\left(\mathbb{R}^{2}\right)}^{p-1-\frac{r_{3}-2}{r_{3}}}\right\| u-v\left\|_{L^{2}\left(\mathbb{R}^{2}\right)}^{\frac{r_{3}-2}{r_{3}}}\right\|_{L^{q_{3}^{\prime}(0, T)}} \\
& \leq\|u\|_{L^{q_{3}^{\prime}\left(p-1-\frac{r_{3}-2}{r_{3}}\right)}\left(0, T ; L^{\infty}\left(\mathbb{R}^{2}\right)\right)}^{p-1-\frac{r_{3}-2}{r_{3}}}\|u\|_{L^{\infty}\left(0, T ; L^{2}\left(\mathbb{R}^{2}\right)\right)}^{\frac{r_{3}-2}{r_{3}}}\|u-v\|_{L^{\infty}\left(0, T ; H^{s_{4}}\left(\mathbb{R}^{2}\right)\right)} \\
& +\|v\|_{L^{\infty}\left(0, T ; H^{\left.s_{4}\left(\mathbb{R}^{2}\right)\right)} \|\right.}\|u-v\|_{L^{q_{3}^{\prime}\left(p-1-\frac{r_{3}-2}{r_{3}}\right)}\left(0, T ; L^{\infty}\left(\mathbb{R}^{2}\right)\right)}^{p-1-\frac{r_{3}-2}{r_{3}}}\|u-v\|_{L^{\infty}\left(0, T ; L^{2}\left(\mathbb{R}^{2}\right)\right)}^{\frac{r_{3}-2}{r_{3}}} .
\end{aligned}
$$

Since

$$
\begin{aligned}
& \|\Phi(u)-\Phi(v)\|_{L^{\infty}\left(0, T ; H^{s}\left(\mathbb{R}^{2}\right)\right)} \\
& \lesssim\left\|u_{0}-v_{0}\right\|_{H^{s}\left(\mathbb{R}^{2}\right)}+\left(\left\|u_{0}\right\|_{H^{s}\left(\mathbb{R}^{2}\right)}+\left\|v_{0}\right\|_{H^{s}\left(\mathbb{R}^{2}\right)}\right)\|u-v\|_{L^{p-1}\left(0, T ; L^{\infty}\left(\mathbb{R}^{2}\right)\right)}^{p-1}
\end{aligned}
$$

the solution map is also continuously dependent in $L^{\infty}\left(0, T ; H^{s}\left(\mathbb{R}^{2}\right)\right)$.

Global well-posedness When $s=1$ and when $s=2$ and $p=3$, a priori estimates shows the global well-posedness by the blow-up alternative argument. Here we consider the case where $p=3$ and $1<s<2$. Let $[a]$ 
be the highest integer which is less than or equal to $a$. Let $T_{1}=\min \left(1, T_{0}\right)$. By using the $H^{1}$ a priori estimate, for any $t>0$,

$$
\begin{aligned}
\|u\|_{L^{4}\left(0, t ; L^{\infty}\left(\mathbb{R}^{2}\right)\right)} & \leq \sum_{k=0}^{\left[t / T_{1}\right]+1}\|u\|_{L^{4}\left(k T_{1},(k+1) T_{1} ; L^{\infty}\left(\mathbb{R}^{2}\right)\right)} \\
& \leq \sum_{k=0}^{\left[t / T_{1}\right]+1}\|u\|_{X^{1}\left(k T_{1},(k+1) T_{1}\right)} \\
& \leq 2 T_{1}^{-1}(1+t)\left\|u_{0}\right\|_{H^{1}\left(\mathbb{R}^{2}\right) .}
\end{aligned}
$$

Then by using Proposition 2.3 ,

$$
\begin{aligned}
\|u(t)\|_{\dot{H}^{s}\left(\mathbb{R}^{2}\right)}^{2} & \lesssim\left\|u_{0}\right\|_{H^{s}\left(\mathbb{R}^{2}\right)}^{2}+\int_{0}^{t}\left\|u\left(t^{\prime}\right)\right\|_{L^{\infty}\left(\mathbb{R}^{n}\right)}^{p-1}\left\|u\left(t^{\prime}\right)\right\|_{\dot{H}^{s}\left(\mathbb{R}^{2}\right)}^{2} d t \\
& \lesssim\left\|u_{0}\right\|_{H^{s}\left(\mathbb{R}^{2}\right)}^{2}+\left\|u\left(t^{\prime}\right)\right\|_{L^{2(p-1)}\left(0, t ; L^{\infty}\left(\mathbb{R}^{2}\right)\right)}^{p-1}\|u\|_{L^{4}\left(0, t ; \dot{H}^{s}\left(\mathbb{R}^{2}\right)\right)}^{2} \\
& \lesssim\left\|u_{0}\right\|_{H^{s}\left(\mathbb{R}^{2}\right)}^{2}+\left\|u_{0}\right\|_{H^{1}\left(\mathbb{R}^{2}\right)}^{p-1}(1+t)^{p-1} t^{\frac{1}{2}-\frac{p-1}{4}}\|u\|_{L^{4}\left(0, t ; \dot{H}^{s}\left(\mathbb{R}^{2}\right)\right)}^{2}
\end{aligned}
$$

This shows

$$
\|u(t)\|_{\dot{H}^{s}\left(\mathbb{R}^{2}\right)}^{4} \lesssim\left\|u_{0}\right\|_{H^{s}\left(\mathbb{R}^{2}\right)}^{4}+\left\|u_{0}\right\|_{H^{1}\left(\mathbb{R}^{2}\right)}^{2(p-1)}(1+t)^{2(p-1)} t^{1-\frac{p-1}{2}}\|u\|_{L^{4}\left(0, t ; \dot{H}^{s}\left(\mathbb{R}^{2}\right)\right)}^{4} .
$$

By the Gronwall inequality,

$$
\|u(t)\|_{H^{s}\left(\mathbb{R}^{2}\right)} \lesssim\left\|u_{0}\right\|_{H^{s}\left(\mathbb{R}^{2}\right)} \exp \left\{C\left\|u_{0}\right\|_{H^{1}\left(\mathbb{R}^{2}\right)}^{2(p-1)}(1+t)^{2(p-1)} t^{2-\frac{p-1}{2}}\right\} .
$$

This shows the global well-posedness in $H^{s}\left(\mathbb{R}^{2}\right)$ setting.

3.3. The case where $n \geq 3$, global $H^{1}$ existence result. In the case where $n \geq 3$, the Strichartz estimate Lemma 3.1 doesn't seem sufficient to obtain a uniform control of solutions in the $H^{1}\left(\mathbb{R}^{3}\right)$ setting. So here, we consider radial data and use the following Strauss lemma.

Lemma 3.3 ([SS, Theorems 1,2], CO, Proposition 1]). Let $n \geq 2$ and let $1 / 2<s<n / 2$. Then

$$
\left\||x|^{\frac{n}{2}-s} f\right\|_{L_{\text {rad }}^{\infty}\left(\mathbb{R}^{n}\right)} \lesssim\|f\|_{\dot{H}_{\text {rad }}^{s}\left(\mathbb{R}^{n}\right)} .
$$

Since solutions are not uniformly controlled at the origin by the Strauss lemma above, we apply the following weighted Strichartz estimate:

Lemma 3.4 ([BGN, Propositions 1.2 and 1.3]). Let $n \in \mathbb{N}$. Let $\delta>0$ and $[x]_{\delta}=|x|^{1-\delta}+|x|^{1+\delta}$. The for any $q_{1} \in[2, \infty)$ and $q_{2} \in(2, \infty)$,

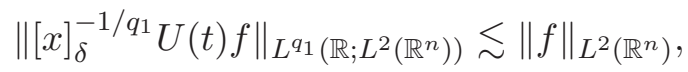

$$
\begin{aligned}
& \left\|[x]_{\delta}^{-1 / q_{1}} \int_{0}^{t} U\left(t-t^{\prime}\right) F\left(t^{\prime}\right) d t^{\prime}\right\|_{L^{q_{1}\left(0, T ; L^{2}\left(\mathbb{R}^{n}\right)\right)}} \lesssim\left\|[x]_{\delta}^{1 / q_{2}} F\right\|_{L^{q_{2}^{\prime}\left(0, T ; L^{2}\left(\mathbb{R}^{n}\right)\right)}} .
\end{aligned}
$$

Proof of Proposition 1.3. By using the uniform $H^{1}\left(\mathbb{R}^{n}\right)$ control obtained in (5)), we reduce the proof to the local well-posedness in $H^{1}\left(\mathbb{R}^{n}\right)$. Let $\delta>0$, $1 / 2<s<1$, and $2<q_{1}, q_{2}<\infty$ satisfy

$$
-(p-1)\left(\frac{n}{2}-s\right)+\frac{1-\delta}{q_{2}}=-\frac{1-\delta}{q_{1}} .
$$


We remark that there exist $\delta, q_{1}, q_{2}, r$ if $1<p<1+2 /(n-2)$ since,

$$
(p-1)\left(\frac{n}{2}-s\right)<1 \Longrightarrow p<1+\frac{2}{n-2 s}<1+\frac{2}{n-2} \text {. }
$$

We define the norm $Y^{1}(T)$ as

$$
\begin{aligned}
\|u\|_{Y^{1}(T)} & =\|u\|_{L^{\infty}\left(0, T ; H_{\mathrm{rad}}^{1}\left(\mathbb{R}^{n}\right)\right)} \\
& +\left\|[x]_{\delta}^{-1 / q_{1}} u\right\|_{L^{q_{1}}\left(0, T ; L_{\mathrm{rad}}^{2}\left(\mathbb{R}^{n}\right)\right)}+\left\|[x]_{\delta}^{-1 / q_{1}} \nabla u\right\|_{L^{q_{1}}\left(0, T ; L_{\mathrm{rad}}^{2}\left(\mathbb{R}^{n}\right)\right)} .
\end{aligned}
$$

Let $\psi \in \mathcal{S}\left(\mathbb{R}^{n}\right)$ satisfy

$$
\psi(x)=\left\{\begin{array}{lll}
1 & \text { if } & |x| \leq 1 \\
0 & \text { if } & |x| \geq 2 .
\end{array}\right.
$$

Then by Lemmas 3.3 and 3.4 and (10),

$$
\begin{aligned}
& \|\Phi(u)\|_{Y^{1}(T)} \\
& \lesssim\left\|u_{0}\right\|_{H_{\mathrm{rad}}^{1}\left(\mathbb{R}^{n}\right)}+\left\|[x]_{\delta}^{-1 / q_{1}} \int_{0}^{t} U\left(t-t^{\prime}\right)\left(\psi\left|u\left(t^{\prime}\right)\right|^{p-1} u\left(t^{\prime}\right)\right) d t^{\prime}\right\|_{Y^{1}(T)} \\
& +\left\|[x]_{\delta}^{-1 / q_{1}} \int_{0}^{t} U\left(t-t^{\prime}\right)\left((1-\psi)\left|u\left(t^{\prime}\right)\right|^{p-1} u\left(t^{\prime}\right)\right) d t^{\prime}\right\|_{Y^{1}(T)} \\
& \lesssim\left\|u_{0}\right\|_{H_{\mathrm{rad}}^{1}\left(\mathbb{R}^{n}\right)} \\
& +\left.\left.\left\|\left.|| x\right|^{-(p-1)\left(\frac{n}{2}-s\right)+\frac{1-\delta}{q_{2}}}\right\| x\right|^{\frac{n}{2}-s} u\right|^{p-1} u \|_{L^{q_{2}^{\prime}}\left(0, T ; L_{\mathrm{rad}}^{2}(|x| \leq 2)\right)} \\
& +\left.\left.\left\||x|^{-(p-1)\left(\frac{n}{2}-s\right)+\frac{1-\delta}{q_{2}}}\right\| x\right|^{\frac{n}{2}-s} u\right|^{p-1} \nabla u \|_{L^{q_{2}^{\prime}}\left(0, T ; L_{\mathrm{rad}}^{2}(|x| \leq 2)\right)} \\
& +\left\||u|^{p-1} u\right\|_{L^{1}\left(0, T ; L_{\mathrm{rad}}^{2}(|x|>1)\right)}+\left\|\nabla\left(|u|^{p-1} u\right)\right\|_{L^{1}\left(0, T ; L_{\mathrm{rad}}^{2}(|x|>1)\right)} \\
& \lesssim\left\|u_{0}\right\|_{H_{\mathrm{rad}}^{1}\left(\mathbb{R}^{n}\right)}+T^{1-\frac{1}{q_{1}}-\frac{1}{q_{2}}\|u\|_{Y^{1}(T)}^{p}}
\end{aligned}
$$

and therefore for some $T$ and $R, \Phi$ is a map from $B_{Y^{1}(T)}(R)$ into itself. Moreover,

$$
\begin{aligned}
& \|\Phi(u)-\Phi(v)\|_{Y^{1}(T)} \\
& \lesssim\left\|u_{0}-v_{0}\right\|_{H_{\mathrm{rad}}^{1}\left(\mathbb{R}^{n}\right)} \\
& +|||x|^{-\frac{1-\delta}{q_{1}}}\left(\left.\left.|| x\right|^{\frac{n}{2}-s} u\right|^{p-1}-\left.\left.|| x\right|^{\frac{n}{2}-s} v\right|^{p-1}\right)(|\nabla u|+|u|) \|_{L^{q_{2}^{\prime}}\left(0, T ; L_{\mathrm{rad}}^{2}(|x| \leq 2)\right)} \\
& +\left.\left.|||x|^{-\frac{1-\delta}{q_{1}}}|| x\right|^{\frac{n}{2}-s} v\right|^{p-1}(|\nabla(u-v)|+|u-v|) \|_{L^{q_{2}^{\prime}}\left(0, T ; L_{\mathrm{rad}}^{2}(|x| \leq 2)\right)} \\
& +\left\|\left(\left.\left.|| x\right|^{\frac{n}{2}-s} u\right|^{p-1}-\left.\left.|| x\right|^{\frac{n}{2}-s} v\right|^{p-1}\right)|x|^{-\frac{1+\delta}{q_{1}}}(|\nabla u|+|u|)\right\|_{L^{1}\left(0, T ; L_{\mathrm{rad}}^{2}(|x|>1)\right)} \\
& +\left.\left.|||| x\right|^{\frac{n}{2}-s} v\right|^{p-1}|x|^{-\frac{1+\delta}{q_{1}}}(|\nabla(u-v)|+|u-v|) \|_{L^{1}\left(0, T ; L_{\mathrm{rad}}^{2}(|x|>1)\right) .}
\end{aligned}
$$

Then for $p \geq 2, \Phi$ is a contraction map on $B_{Y^{1}(T)}(R)$. Similarly, for $1<$ $p<2$, we define the auxiliary norm $Y^{0}(T)$ as

$$
\|u\|_{Y^{0}(T)}=\|u\|_{L^{\infty}\left(0, T ; L_{\mathrm{rad}}^{2}\left(\mathbb{R}^{n}\right)\right)}+\left\|[x]_{\delta}^{-1 / q_{1}} u\right\|_{L^{q}\left(0, T ; L_{\mathrm{rad}}^{2}\left(\mathbb{R}^{n}\right)\right)} .
$$


Then for $1<p<2$,

$$
\begin{aligned}
& \|(\Phi(u)-\Phi(v))\|_{Y^{0}(T)} \\
& \lesssim\left\|u_{0}-v_{0}\right\|_{L^{2}\left(\mathbb{R}^{n}\right)} \\
& +\left\|[x]_{\delta}^{-1 / q_{1}}\left(\left.|| x\right|^{\frac{n}{2}-s} v|+||x|^{\frac{n}{2}-s} v \mid\right)^{p-1}|u-v|\right\|_{L^{q_{2}^{\prime}\left(0, T ; L_{\mathrm{rad}}^{2}(|x| \leq 2)\right)}} \\
& +\left\|\left(\left.|| x\right|^{\frac{n}{2}-s} v|+||x|^{\frac{n}{2}-s} v \mid\right)^{p-1}|u-v|\right\|_{L^{1}\left(0, T ; L_{\mathrm{rad}}^{2}(|x|>1)\right)} \\
& \lesssim T^{1-\frac{1}{q_{2}}}\left(\|u\|_{Y^{1}(T)}+\|v\|_{Y^{1}(T)}\right)^{p-1}\|u-v\|_{Y^{0}(T)} .
\end{aligned}
$$

and therefore $\Phi$ is a contraction map in $Y^{0}(T)$ for some $T$ and $R$ and therefore we have a unique solution to (1) in $Y^{1}(T)$. Moreover, by Lemma 3.3 and (11), with some $0<\theta<1$,

$$
\begin{aligned}
& \|u-v\|_{Y^{1}(T)} \\
& \lesssim\left\|u_{0}-v_{0}\right\|_{H_{\text {rad }}^{1}\left(\mathbb{R}^{n}\right)}+T^{1-\frac{1}{q_{1}}-\frac{1}{q_{2}}}\left(\|u\|_{Y^{1}(T)}+\|v\|_{Y^{1}(T)}\right)^{p-1}\|u-v\|_{Y^{1}(T)} \\
& +T^{1-\frac{1}{q_{1}}-\frac{1}{q_{2}}}\left(\|u\|_{Y^{1}(T)}+\|v\|_{Y^{1}(T)}\right)\left\||x|^{\frac{n}{2}-s}(u-v)\right\|_{L^{\infty}\left(0, T ; L_{\text {rad }}^{\infty}\left(\mathbb{R}^{n}\right)\right.}^{p-1} \\
& \lesssim\left\|u_{0}-v_{0}\right\|_{H_{\mathrm{rad}}^{1}\left(\mathbb{R}^{n}\right)}+T^{1-\frac{1}{q_{1}}-\frac{1}{q_{2}}}\left(\|u\|_{Y^{1}(T)}+\|v\|_{Y^{1}(T)}\right)^{p-1}\|u-v\|_{Y^{1}(T)} \\
& +T\left(\|u\|_{Y^{1}(T)}+\|v\|_{Y^{1}(T)}\right)\|u-v\|_{Y^{0}(T)}^{p-1}
\end{aligned}
$$

and therefore $\|u-v\|_{Y^{1}(T)} \rightarrow 0$ as $\left\|u_{0}-v_{0}\right\|_{H_{\mathrm{rad}}^{1}\left(\mathbb{R}^{n}\right)} \rightarrow 0$.

3.4. 3 dimensional case, small $H^{1}$ data solutions for $p=3$. In the three dimensional scaling critical case, the weighted Strichartz estimate Lemma 3.4 doesn't seem sufficient to control solutions uniformly. So here, we transform (11) into the corresponding wave equation.

The Cauchy problem (11) with initial data $u(0)=u_{0}$ is rewritten as the following:

$$
\begin{aligned}
\square u & =-i\left(-i \partial_{t}-D\right)|u|^{p-1} u \\
& =i \frac{p+1}{2}|u|^{p-1}\left(D u-i|u|^{p-1} u\right) \\
& -i \frac{p-1}{2}|u|^{p-3} u^{2} \overline{\left(D u-i|u|^{p-1} u\right)}+i D\left(|u|^{p-1} u\right) \\
& -i\left(D\left(|u|^{p-1} u\right)+\frac{p+1}{2}|u|^{p-1} D u-\frac{p-1}{2}|u|^{p-3} u^{2} D \bar{u}\right)+p|u|^{2 p-2} u \\
& =: F_{p}(u) .
\end{aligned}
$$

Then the corresponding integral equation is the following:

$$
\begin{aligned}
u(t) & =\cos (t D) u_{0}+\frac{\sin (t D)}{D}\left(-i D u_{0}-\left|u_{0}\right|^{p-1} u_{0}\right) \\
& +\int_{0}^{t} \frac{\sin \left(\left(t-t^{\prime}\right) D\right)}{D} F_{p}(u)\left(t^{\prime}\right) d t^{\prime} .
\end{aligned}
$$


For any radially symmetric function $f$, we define $\tilde{f}$ as $\widetilde{f}(|x|)=f(x)$. Then for any radial data, (12) is rewritten as

(13) $\widetilde{u}(t)$

$$
=\partial_{t} J\left[u_{0}\right](t)+J\left[-i D u_{0}-\left|u_{0}\right|^{p-1} u_{0}\right](t)+\int_{0}^{t} J\left[F_{p}(u)\left(t^{\prime}\right)\right]\left(t-t^{\prime}\right) d t^{\prime}
$$

where

$$
J[f](t, r)=\frac{1}{2 r} \int_{|r-t|}^{r+t} \lambda \widetilde{f}(\lambda) d \lambda .
$$

This transformation is justified as follows:

Lemma 3.5. Let $1<p \leq 3$ and $u_{0} \in H_{\mathrm{rad}}^{1}\left(\mathbb{R}^{3}\right)$ and $u \in C\left(0, T ; H_{\mathrm{rad}}^{1}\left(\mathbb{R}^{3}\right)\right)$ be the solution of (4). Then $u$ is also the solution of (13)

Proof. Since $H^{1}\left(\mathbb{R}^{3}\right) \hookrightarrow L^{2 p}\left(\mathbb{R}^{3}\right), u \in C^{1}\left(0, T ; L_{\mathrm{rad}}^{2}\left(\mathbb{R}^{3}\right)\right)$ and $|u|^{p-1} u \in$ $C^{1}\left(0, T ; H_{\text {rad }}^{s}\left(\mathbb{R}^{3}\right)\right)$ with $s<-3 / 2$. Then $-i\left(-i \partial_{t}-D\right)|u|^{p-1} u=F_{p}(u)$ holds in the $H_{\text {rad }}^{s}\left(\mathbb{R}^{3}\right)$ setting. Moreover,

$$
U(t) u_{0}=\cos (t D) u_{0}-i \sin (t D) u_{0}
$$

and in the $H_{\text {rad }}^{s}\left(\mathbb{R}^{3}\right)$ setting, the following calculation is also justified:

$$
\begin{aligned}
& -\int_{0}^{t} U\left(t-t^{\prime}\right)\left(\left|u\left(t^{\prime}\right)\right|^{p-1} u\left(t^{\prime}\right)\right) d t^{\prime} \\
= & -\int_{0}^{t} \cos \left(\left(t-t^{\prime}\right) D\right)\left(\left|u\left(t^{\prime}\right)\right|^{p-1} u\left(t^{\prime}\right)\right) d t^{\prime} \\
& -\int_{0}^{t}-i \sin \left(\left(t-t^{\prime}\right) D\right)\left(\left|u\left(t^{\prime}\right)\right|^{p-1} u\left(t^{\prime}\right)\right) d t^{\prime} \\
= & {\left[\frac{\sin \left(\left(t-t^{\prime}\right) D\right)}{D}\left(\left|u\left(t^{\prime}\right)\right|^{p-1} u\left(t^{\prime}\right)\right)\right]_{t^{\prime}=0}^{t} } \\
- & \int_{0}^{t} \frac{\sin \left(\left(t-t^{\prime}\right) D\right)}{D} \partial_{t}\left(\left|u\left(t^{\prime}\right)\right|^{p-1} u\left(t^{\prime}\right)\right) d t^{\prime} \\
- & \int_{0}^{t}-i \sin \left(\left(t-t^{\prime}\right) D\right)\left(\left|u\left(t^{\prime}\right)\right|^{p-1} u\left(t^{\prime}\right)\right) d t^{\prime} \\
= & -\frac{\sin (t D)}{D}\left(\left|u_{0}\right|^{p-1} u_{0}\right)-i \int_{0}^{t} \frac{\sin \left(\left(t-t^{\prime}\right) D\right)}{D}\left(-i \partial_{t}-D\right)\left(\left|u\left(t^{\prime}\right)\right|^{p-1} u\left(t^{\prime}\right)\right) d t^{\prime}
\end{aligned}
$$

Therefore $u$ is also a solution of (13).

To obtain the uniform control, we use the estimates below regarding $J$. For any $f:[0, \infty) \rightarrow \mathbb{C}$, we define $A[f]: \mathbb{R} \rightarrow \mathbb{C}$ as $A[f](\lambda)=f(|\lambda|)$. See also $\mathrm{KM}$.

Lemma 3.6. Let $f:[0, \infty) \rightarrow \mathbb{C}$. Then

$$
\left\|\frac{1}{2 \cdot} \int_{|\cdot-t|}^{\cdot+t} f(\lambda) d \lambda\right\|_{L^{\infty}(0, \infty)} \leq M[A[f]](t) .
$$

Proof. If $r \leq t$, then

$$
\frac{1}{2 r} \int_{t-r}^{t+r}|f(\lambda)| d \lambda \leq M[f](t)
$$


If $r>t$, then

$$
\frac{1}{2 r} \int_{r-t}^{t+r}|f(\lambda)| d \lambda \leq \frac{1}{2 r} \int_{t-r}^{t+r}|A[f](\lambda)| d \lambda \leq M[A[f]](t) .
$$

Corollary 3.7. Let $f: \mathbb{R}^{3} \rightarrow \mathbb{C}$ be radial. Then

$$
\|J[f]\|_{L^{2}\left(0, T ; L^{\infty}\left(\mathbb{R}^{3}\right)\right)} \leq C\|f\|_{L_{\mathrm{rad}}^{2}\left(\mathbb{R}^{3}\right)} .
$$

Proof. Let $g(\lambda)=\lambda \widetilde{f}(\lambda)$. Then

$$
\|J[f]\|_{L^{2}\left(0, T ; L^{\infty}(0, \infty)\right)} \leq\|M[A[g]]\|_{L^{2}(0, T)} \leq C\|g\|_{L^{2}(0, \infty)}=C\|f\|_{L_{\mathrm{rad}}^{2}\left(\mathbb{R}^{3}\right)} .
$$

Corollary 3.8. Let $h:[0, \infty) \times \mathbb{R}^{3} \rightarrow \mathbb{C}$ be radial. Then

$$
\left\|\int_{0}^{t} J\left[h\left(t^{\prime}\right)\right]\left(t-t^{\prime}\right) d t^{\prime}\right\|_{L^{2}\left(0, T ; L^{\infty}(0, \infty)\right)} \leq C\|h\|_{L^{1}\left(0, T ; L_{\mathrm{rad}}^{2}\left(\mathbb{R}^{3}\right)\right)} .
$$

Proof. Let $H(t, \lambda)=\lambda \widetilde{h}(t, \lambda)$. Then

$$
\begin{aligned}
\left\|\int_{0}^{t} J\left[h\left(t^{\prime}\right)\right]\left(t-t^{\prime}\right) d t^{\prime}\right\|_{L^{2}\left(0, T ; L^{\infty}(0, \infty)\right)} & \leq\left\|\int_{0}^{t} M\left[A\left[H\left(t^{\prime}\right)\right]\right]\left(t-t^{\prime}\right) d t^{\prime}\right\|_{L^{2}(0, T)} \\
& \leq \int_{0}^{T}\left\|M\left[A\left[H\left(t^{\prime}\right)\right]\right]\right\|_{L^{2}\left(t^{\prime}, T\right)} d t^{\prime} \\
& \leq C \int_{0}^{T}\left\|h\left(t^{\prime}\right)\right\|_{L_{\mathrm{rad}}^{2}\left(\mathbb{R}^{3}\right)} d t^{\prime} .
\end{aligned}
$$

Corollary 3.9 (Hardy). Let $f \in C^{1}(\mathbb{R} ; \mathbb{C})$. Then

$$
\left\|\frac{d}{d t}\left(\frac{1}{2 r} \int_{|r-t|}^{r+t} \lambda f(\lambda) d \lambda\right)\right\|_{L^{2}\left(0, \infty ; L^{\infty}(0, \infty)\right)} \leq C\left\|r f^{\prime}\right\|_{L^{2}(0, \infty)} .
$$

Proof of Proposition 1.4. For $0<T<1$ and $p=3$, By Corollaries 3.7, 3.8, and 3.9 .

$$
\begin{aligned}
& \|u\|_{L^{2}\left(0, T ; L_{\mathrm{rad}}^{\infty}\left(\mathbb{R}^{3}\right)\right)} \\
& \lesssim\left\|u_{0}\right\|_{H_{\mathrm{rad}}^{1}\left(\mathbb{R}^{3}\right)}+\left\|u_{0}\right\|_{H_{\mathrm{rad}}^{1}\left(\mathbb{R}^{3}\right)}^{3}+\left\|F_{3}\right\|_{L^{1}\left(0, T ; L_{\mathrm{rad}}^{2}\left(\mathbb{R}^{3}\right)\right)} \\
& \lesssim\left\|u_{0}\right\|_{H_{\mathrm{rad}}^{1}\left(\mathbb{R}^{3}\right)}+\left\|u_{0}\right\|_{H_{\mathrm{rad}}^{1}\left(\mathbb{R}^{3}\right)}^{3} \\
& +\|u\|_{L^{2}\left(0, T ; L_{\mathrm{rad}}^{\infty}\left(\mathbb{R}^{3}\right)\right.}^{2}\|u\|_{L^{\infty}\left(0, T ; H_{\mathrm{rad}}^{1}\left(\mathbb{R}^{3}\right)\right)}+\left\|\left.u\right|^{5}\right\|_{L^{1}\left(0, T ; L_{\mathrm{rad}}^{2}\left(\mathbb{R}^{3}\right)\right)} .
\end{aligned}
$$

Since

$$
\left\||u|^{5}\right\|_{L^{1}\left(0, T ; L_{\mathrm{rad}}^{2}\left(\mathbb{R}^{3}\right)\right)} \leq\|u\|_{L^{2}\left(0, T ; L_{\mathrm{rad}}^{\infty}\left(\mathbb{R}^{3}\right)\right)}^{2}\|u\|_{L^{\infty}\left(0, T ; H_{\mathrm{rad}}^{1}\left(\mathbb{R}^{3}\right)\right)}^{3},
$$

by the unitarity of $U(t)$,

$$
\|u\|_{L^{\infty}\left(0, T ; H_{\mathrm{rad}}^{1}\left(\mathbb{R}^{3}\right)\right)} \lesssim\left\|u_{0}\right\|_{H_{\mathrm{rad}}^{1}\left(\mathbb{R}^{3}\right)}+\|u\|_{L^{p-1}\left(0, T ; L_{\mathrm{rad}}^{\infty}\left(\mathbb{R}^{3}\right)\right)}^{p-1}\left\|u_{0}\right\|_{H_{\mathrm{rad}}^{1}\left(\mathbb{R}^{3}\right)} .
$$


Let $X_{\mathrm{rad}}^{1}(0, T)=L^{\infty}\left(0, T ; H_{\mathrm{rad}}^{1}\left(\mathbb{R}^{3}\right)\right) \cap L^{2}\left(0, T ; L_{\mathrm{rad}}^{\infty}\left(\mathbb{R}^{3}\right)\right)$. Then, for sufficiently small initial data $u_{0}, \Phi$ maps from $B_{X_{\mathrm{rad}}^{1}(0, T)}(R)$ into itself with some $T$ and $R$. Moreover,

$$
\begin{aligned}
& \left\||u|^{2} u-|v|^{2} v\right\|_{L^{1}\left(0, T ; H_{\mathrm{rad}}^{1}\left(\mathbb{R}^{3}\right)\right)} \\
& \lesssim\left(\|u\|_{X_{\mathrm{rad}}(0, T)}+\|v\|_{X_{\mathrm{rad}}(0, T)}\right)^{2}\|u-v\|_{L^{\infty}\left(0, T ; H_{\mathrm{rad}}^{1}\left(\mathbb{R}^{3}\right)\right)} .
\end{aligned}
$$

Since

$$
\begin{aligned}
& |F(u)-F(v)| \\
& =\left.\left|i\left(D\left(|u|^{2 p-1} u\right)-2|u|^{2} D u-u^{2} D \bar{u}\right)+3\right| u\right|^{4} u \\
& -i\left(D\left(|v|^{2} v\right)-2|v|^{2} D v-v^{2} D \bar{v}\right)-3|v|^{4} v \mid \\
& \leq\left|D\left(|u|^{2} u-|v|^{2} v\right)\right|+|u|^{2}|D(u-v)| \\
& +\left(\left.|| u\right|^{2}-|v|^{2}|+| u^{2}-v^{2} \mid\right)|D v|+\left.3|| u\right|^{4} u-|v|^{4} v \mid,
\end{aligned}
$$

we have

$$
\begin{aligned}
& \|F(u)-F(v)\|_{L^{1}\left(0, T ; L_{\mathrm{rad}}^{2}\left(\mathbb{R}^{3}\right)\right)} \\
& \lesssim\left(\|u\|_{X_{\mathrm{rad}}^{1}(0, T)}+\|v\|_{X_{\mathrm{rad}}^{1}(0, T)}\right)^{2}\|u-v\|_{X_{\mathrm{rad}}^{1}(0, T)} \\
& +\left(\|u\|_{X_{\mathrm{rad}}^{1}(0, T)}+\|v\|_{X_{\mathrm{rad}}^{1}(0, T)}\right)^{4}\|u-v\|_{X_{\mathrm{rad}}^{1}(0, T)} .
\end{aligned}
$$

This means for sufficiently small $u_{0}, \Phi$ is a contraction map on $B_{X_{\mathrm{rad}}^{1}(0, T)}(R)$.

\section{REFERENCES}

[ACGM] P. Acquistapace, A. P. Candeloro, V. Georgiev, and M. L. Manca, Mathematical Phase Model of Neural Populations Interaction in Modulation of REM/NREM Sleep, Math. Model. Anal., 21 (2016), 794-810.

[BGN] Jacopo Bellazzini, Vladimir Georgiev, and Nicola Visciglia, Long time dynamics for semirelativistic NLS and Half Wave in arbitrary dimension, 2016; arXiv:1611.04823.

[C] T. Cazenave, "Semilinear Schrödinger equations," American Mathematical Society, 2003.

[CW1] T. Cazenave and F. B. Weissler. "Some remarks on the nonlinear Schrödinger equation in the subcritical case". In New methods and results in nonlinear field equations (Bielefeld, 1987), volume 347 of Lecture Notes in Phys., 59-69. Springer, Berlin, (1989).

[CW2] T. Cazenave and F. B. Weissler, The Cauchy problem for the critical nonlinear Schrödinger equation in $H^{s}$, Nonlinear Anal., 14 (1990), 807-836.

[CO] Y. Cho and T. Ozawa, Sobolev inequalities with symmetry, Commun. Contemp. Math., 11 (2009), 355 - 365.

[F] K. Fujiwara, Remark on local solvability of the Cauchy problem for semirelativistic equations, J. Math. Anal. Appl., 432 (2015), 744-748.

[GOV] J. Ginibre, T. Ozawa, and G. Velo, On the existence of the wave operators for a class of nonlinear Schrödinger equations, Ann. Inst. H. Poincaré Phys. Théor., 60(1994), $211-239$.

[GV] J. Ginibre and G. Velo, Generalized Strichartz inequalities for the wave equations, J. Funct. Anal., 133 (1995), 50 - 68.

[GW] Z. Guo and Y. Wang, Improved Strichartz estimates for a class of dispersive equations in the radial case and their applications to nonlinear Schrödinger and wave equations, J. Math. Anal., 124(2014), 1 - 38. 
[II] M. Ikeda and T. Inui, Some non-existence results for the semilinear Schrödinger equation without gauge invariance, J. Math. Anal. Appl., 425 (2015), 758-773.

[IW] M. Ikeda and Y. Wakasugi, Small-data blow-up of $L^{2}$-solution for the nonlinear Schrödinger equation without gauge invariance, Differential Integral Equations, 26 (2013), 1275-1285.

[I] T.Inui, Some nonexistence results for a semirelativistic Schrödinger equation with nongauge power type nonlinearity, Proc. Amer. Math. Soc., 144 (2016), $2901-2909$.

[KM] S. Klainerman and M. Machedon, Space-time estimates for null forms and the local existence theorem, Comm. Pure Appl. Math., 46 (1993), 1221 - 1268.

[Kur] Y. Kuramoto, "Chemical Oscillations, Waves and Turbolence," Springer-Verlag, New York, 1984.

[Las] N. Laskin, Fractional quantum mechanics and Levy path integrals, Physics Letters A, 268 (2000), $298-304$.

[NO] M. Nakamura and T. Ozawa, The Cauchy problem for nonlinear Klein-Gordon equations in the Sobolev spaces, Publ. Res. Inst. Math. Sci., 37 (2001), 255 - 293.

[O] T. Ozawa, Remarks on proofs of conservation laws for nonlinear Schrödinger equations, Calc. Var. Partial Differential Equations, 25 (2006), 403 - 408.

[SS] W. Sickel and L. Skrzypczak, Radial subspaces of Besov and Lizorkin-Triebeel classes: extended Strauss lemma and compactness of embedding, J. Fourier Anal. Appl. 6(2000), $639-662$.

Department of Pure and Applied Physics, Waseda University, 3-4-1, Okubo, SHINJUKU-KU, TOKYO 169-8555, JAPAN

E-mail address: k-fujiwara@asagi.waseda.jp

Department of Mathematics, University of Pisa, Largo Bruno Pontecorvo 5 I - 56127 Pisa, Italy, and, Faculty of Science and Engineering, Waseda UniVersity, 3-4-1, Okubo, ShinJuku-Ku, TOKYo 169-8555, JAPAN

E-mail address: georgiev@dm.unipi.it

Department of Applied Physics, Waseda University, 3-4-1, Okubo, ShinjukuKU, TOKYO 169-8555, JAPAN

E-mail address: txozawa@waseda.jp 